\title{
The Saliva of Probands Sucking an lota-Carrageenan Containing Lozenge Inhibits Viral Binding and Replication of the Most Predominant Common Cold Viruses and SARS-CoV-2
}

\author{
Martina Morokutti-Kurz' \\ Nicole Unger-Manhart ${ }^{\prime}$ \\ Philipp Graf' \\ Pia Rauch ${ }^{2}$ \\ Julia Kodnar' \\ Maximilian Große ${ }^{2}$ \\ Christian Setz ${ }^{2}$ \\ Markus Savli ${ }^{3}$ \\ Friedrich Ehrenreich ${ }^{4}$ \\ Andreas Grassauer' \\ Eva Prieschl-Grassauer (iD) \\ Ulrich Schubert ${ }^{2}$ \\ 'Marinomed Biotech AG, Korneuburg, \\ 2100, Austria; ${ }^{2}$ Institute of Virology, \\ Friedrich-Alexander University Erlangen- \\ Nürnberg (FAU), Erlangen, Germany; \\ ${ }^{3}$ Biostatistik \& Consulting Savli, Hartberg, \\ 8230, Austria; ${ }^{4}$ Practice Dr. Friedrich \\ Ehrenreich, Vienna, II70, Austria
}

Correspondence: Martina MorokuttiKurz

Marinomed Biotech AG, Korneuburg, 2100 , Austria

Email martina.morokutti-

kurz@marinomed.com

Ulrich Schubert

Institute of Virology, Friedrich-Alexander University Erlangen-Nürnberg (FAU),

Erlangen, Germany

Email ulrich.schubert@fau.de
Purpose: The aim of this study was to investigate whether sucking of an iota-carrageenan containing lozenge releases sufficient iota-carrageenan into the saliva of healthy subjects to neutralize representatives of the most common respiratory virus families causing common cold and SARS-CoV-2.

Patients and Methods: In this monocentric, open label, prospective clinical trial, 31 healthy subjects were included to suck a commercially available iota-carrageenan containing lozenge. Saliva samples from 27 subjects were used for ex vivo efficacy analysis. The study's primary objective was to assess if the mean iota-carrageenan concentration of the saliva samples exceeded $5 \mu \mathrm{g} / \mathrm{mL}$, which is the concentration known to reduce replication of human rhinovirus (hRV) 1a and 8 by $90 \%$. The iota-carrageenan concentration of the saliva samples was analyzed by UV-Vis spectroscopy. The antiviral effectiveness of the individual saliva samples was determined in vitro against a panel of respiratory viruses including hRV1a, hRV8, human coronavirus OC43, influenza virus A H1N1pdm09, coxsackievirus A10, parainfluenza virus 3 and SARS-CoV-2 using standard virological assays.

Results: The mean iota-carrageenan concentration detected in the saliva exceeds the concentration needed to inhibit $90 \%$ of hRV1a and hRV 8 replication by 134 -fold (95\% CI 116.3-160.8-fold; $\mathrm{p}<0.001$ ). Thus, the study met the primary endpoint. Furthermore, the iota-carrageenan saliva concentration was 60 to 30,351-fold higher than needed to reduce viral replication/binding of all tested viruses by at least $90 \%(\mathrm{p}<0.001)$. The effect was most pronounced in hCoV OC43; in case of SARS-CoV-2, the $\mathrm{IC}_{90}$ was exceeded by 121 -fold ( $\left.\mathrm{p}<0.001\right)$.

Conclusion: Sucking an iota-carrageenan containing lozenge releases sufficient iota-carrageenan to neutralize and inactivate the most abundant respiratory viruses as well as pandemic SARS-CoV-2. The lozenges are therefore an appropriate measure to reduce the viral load at the site of infection, hereby presumably limiting transmission within a population as well as translocation to the lower respiratory tract.

Trial Registration: NCT04533906.

Keywords: iota-carrageenan, respiratory viruses, corona virus, lozenges, antiviral, clinical study, SARS-CoV-2

\section{Introduction}

Respiratory tract infections (RTIs) are among the most common diseases to affect humans with typical symptoms such as rhinitis, cough, fever, and sore throat ${ }^{1}$ and, in worst case, severe respiratory syndromes. Most of these infections are caused by 
viruses, predominately human rhinoviruses and endemic coronaviruses, followed by ortho- and paramyxoviruses (influenza virus, parainfluenza virus) and adenoviruses. ${ }^{2}$ Currently, the global spread of SARS-CoV-2/COVID-19 is devastating health systems and economies worldwide.

Respiratory viruses enter the body mainly via the nasopharyngeal cavity. They do not only infect the nasal epithelium but also other parts of the upper respiratory tract such as the pharyngeal mucosa. From there, infection may spread to the lower respiratory tract, causing severe lung infection. RTIs are transmitted from person to person by respiratory droplets or indirectly via contaminated surfaces. Reducing the viral load at the site of infection presumably limits the risk of transmission within a population and/or the individual's respiratory tract.

Treatment of respiratory viral infection with iota-carrageenan nasal spray has already shown to be safe and effective in five randomized, double blind, placebo-controlled clinical trials with more than 600 children and adults suffering from respiratory viral infection. Therapeutic application of an iota-carrageenan nasal spray reduced the viral load which also manifested clinically by reducing the severity and duration of symptoms as well as the number of relapses in the verum group. ${ }^{3-7}$ Recently it was shown that an iota-carrageenan containing nasal spray showed a significant prophylactic efficacy in preventing SARS-CoV-2 infection in healthcare personnel caring for patients with COVID-19 disease. ${ }^{8}$

Carrageenan is a high molecular weight sulfated polymer derived from red seaweed (Rhodophyceae) that has been extensively used in food, cosmetic and pharmaceutical industry and is generally recognized as safe by the FDA (GRAS). Three main forms of carrageenans are commercially used: iota, kappa and lambda. They differ from each other in the degree of sulfation, solubility and gelling properties. ${ }^{20}$ The antiviral activity of iota-carrageenan is well-established and has been demonstrated in vitro and in vivo with a variety of respiratory viruses including SARS-CoV-2. ${ }^{9-16}$ The mode of action of iota-carrageenan is solely physical. Once at the site of infection at an effective concentration, it covers the viral surface by rather indiscriminate electrostatic interactions, thereby preventing its binding to the mucosa, internalization and, as a result, primary infection. In case that infection has already taken place, newly synthesized virus particles that are excreted from the cells are neutralized by iota-carrageenan as well, resulting in an inhibition of viral spread. Locally acting lozenges are known to reach the site of infection directly. It is therefore legitimate to hypothesize that they thereby exert maximal efficacy. We already published that dissolved lozenges containing $10 \mathrm{mg}$ iotacarrageenan were highly active in binding and inactivating hRV1a and hRV8, coxsackievirus A10, hCoV OC43 and influenza virus A H1N1n. ${ }^{11}$

The aim of this study was to investigate whether the iota-carrageenan that is released into the saliva of subjects upon sucking a lozenge containing $10 \mathrm{mg}$ iota-carrageenan is sufficient to inhibit the replication of the most common respiratory viruses causing common cold and sore throat: hRV1a and 8, hCoV OC43, influenza virus A H1N1n, parainfluenza virus 3 and coxsackievirus $A 10$. In the light of the current SARS-CoV-2 pandemic, the samples were also tested for their potential to inhibit the replication of SARS-CoV-2.

\section{Materials and Methods Study Design}

This post-marketing, monocentric, open label clinical study was conducted according to MPG $\S 40 / 3$ at a general practitioner in Vienna during July 2020. The study was performed in compliance with the Declaration of Helsinki and the Guideline for Good Clinical Practice, and all applicable local laws and regulations. The protocol received clearance from the Austrian Competent Authority as well as the ethics committee (EK 19-295-0120) of the City of Vienna. After receiving informed consent, 31 eligible male and non-pregnant female subjects with no signs of an acute respiratory infection were included. Subjects had to collect native saliva before sucking one lozenge with an iota-carrageenan content of $10 \mathrm{mg}$. During sucking of the lozenge subjects donated their saliva instead of swallowing it. When the collected saliva volume reached about $5 \mathrm{~mL}$ sucking was terminated and samples were stored at $-20^{\circ} \mathrm{C}$.

Four saliva samples had to be excluded from analysis since blood traces were visible due to periodontitis of the participants as stated by the principal investigator (Figure 1).

\section{Study Medication}

Coldamaris $^{\circledR}$ iota-carrageenan Lozenges (Lot $\mathrm{Nr}$. 8030007).

\section{Sample Processing}

To obtain homogenous and reproducible baseline results, native saliva samples from 27 subjects were pooled whereas 


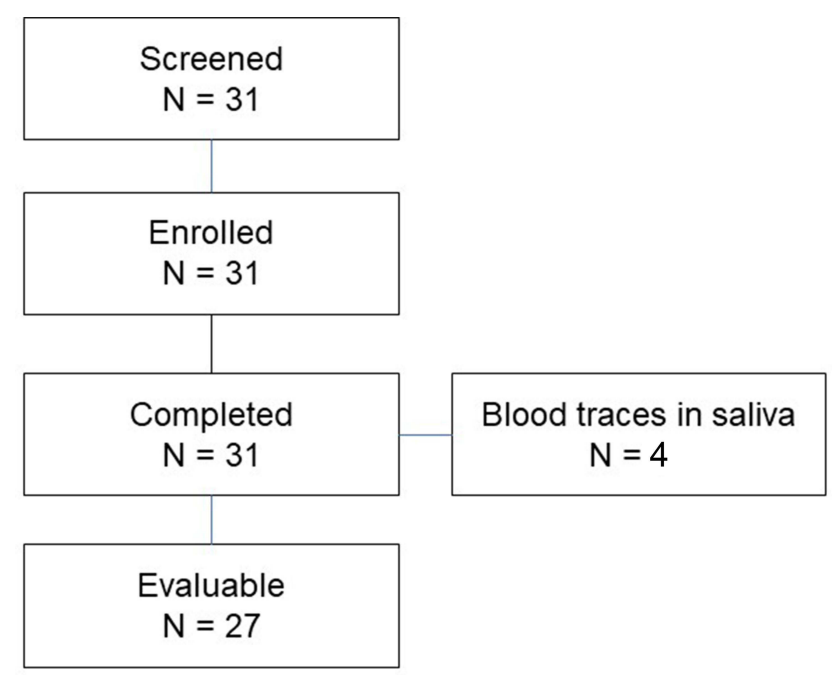

Figure I Distribution of subjects.

treated saliva samples were analyzed separately. All samples were diluted 1:2 with cell culture water, pasteurized at $80^{\circ} \mathrm{C}$ for $35 \mathrm{~min}$ to ensure safe handling of the samples. Finally, samples were aliquoted. One aliquot was used for determination of carrageenan concentration, and the remaining aliquots were used in the antiviral activity assays. The pasteurization process did not affect the sample quality and the carrageenan content (data not shown).

\section{Evaluation of Efficacy}

The primary objective was to determine whether the mean iota-carrageenan concentration in saliva during sucking an iota-carrageenan containing lozenge reached known $\mathrm{IC}_{90}$ values for $\mathrm{hRV} 1 \mathrm{a}$ and $\mathrm{hRV} 8(5 \mu \mathrm{g} / \mathrm{mL}) .{ }^{9}$ The primary efficacy variable was defined as the mean iota-carrageenan concentration in saliva during sucking an iota-carrageenan containing lozenge.

The secondary objectives were to determine whether the mean iota-carrageenan concentration in treated saliva $\left(\mu \mathrm{g} / \mathrm{mL}\right.$; base line corrected) reached the respective $\mathrm{IC}_{90} /$ MIC values (paired t-tests) of the saliva samples for hRV1a, hRV8, hCoV OC43, influenza virus A H1N1n, coxsackievirus A10, PIV3 and SARS-CoV-2. The secondary efficacy variable was defined as the specific antiviral activity of the treated saliva samples against a set of respiratory viruses expressed as inhibitory concentration $50 \%$ or $90 \%\left(\mathrm{IC}_{50}, \mathrm{IC}_{90}\right)$ or as minimal inhibitory concentration (MIC). These virological parameters were determined in vitro using standard virological assays (described later).

\section{Determination of the lota-Carrageenan Concentration}

The iota-carrageenan concentration of the heat-inactivated samples was determined by means of UV/Vis detection after complex formation with methylene blue (MB). ${ }^{6}$ Briefly, methylene blue interacts with iota-carrageenan at low concentrations to form a water-soluble meta-chromic complex. The complexation results in a change of the dye from blue (absorptions maxima at 610 and $666 \mathrm{~nm}$ ) to purple with a maximum absorption wavelength of $559 \mathrm{~nm}$. The absorbance values at these wavelengths are proportional to the iota-carrageenan concentration. Also, pooled native samples were tested accordingly. The measured iota-carrageenan concentrations of the treated samples were corrected with the signal of the native samples (baseline corrected) to avoid overestimation of the iota-carrageenan concentration of the treated samples.

\section{Determination of the Antiviral Activity}

A sample of known inhibitory activity (iota-carrageenan in $0.5 \% \mathrm{NaCl})$, a negative control $(0.5 \% \mathrm{NaCl}$ and/or untreated saliva) and toxicity/agglutination controls in the absence of virus were included in every assay to ensure that the assay was functional and to relate the activity of any treated saliva sample to a reference of known effectiveness. The assays were standardized and only assays meeting the predefined acceptance criteria were used for data evaluation.

\section{Replication Inhibition Assays - hRVIa, hRV8, Coxsackievirus Al0}

Virus and cell cultivation as well as antiviral activity assays for hRV1a, hRV8 and coxsackievirus A10 were performed as previously described. ${ }^{11}$ In short, the respective virus was preincubated with a semilogarithmic dilution series of the respective treated saliva before it was added to permissive cell lines (HeLa/RD) for infection. After infection, cells were washed with medium and cultured at $33^{\circ} \mathrm{C}(\mathrm{hRV})$ or $37^{\circ} \mathrm{C}$ (coxsackievirus A10), hereby maintaining the same dilution of treated saliva as in the prophylactic treatment. The specific antiviral activity was established by determining the effect on cell viability. An incubation of cells with the same dilution series in the absence of viral infection was performed to monitor a potential toxicity of the treatment.

\section{Replication Inhibition Assay - SARS-CoV-2}

Virus isolation, virus and Vero B4 cell cultivation were performed as described elsewhere. ${ }^{18}$ For determining the 
antiviral activity, Vero B4 cells were maintained in Dulbecco's Modified Eagle's Medium (DMEM, Gibco, Carlsbad, USA) containing $10 \%(\mathrm{v} / \mathrm{v})$ inactivated fetal calf serum (FCS, Capricorn Scientific, Ebsdorfergrund, Germany), 2 mM l-glutamine (Gibco, Carlsbad, USA), $100 \mathrm{U} / \mathrm{mL}$ penicillin (Sigma, St. Louis, USA), and 100 $\mu \mathrm{g} / \mathrm{mL}$ streptomycin (Sigma, St. Louis, USA).

Per 24 well plate, $4.8 \times 10^{6}$ Vero B4 cells were resuspended in $3.6 \mathrm{~mL}$ medium and infected in FCS-free DMEM with an MOI of 0.02 of the field isolate SARS-CoV-2PR-1 for $1 \mathrm{~h}$ at $37^{\circ} \mathrm{C}$ under gentle rotation. Afterwards, the inoculum was removed. After washing with PBS, the cells were resuspended in the amount of medium necessary to seed $2 \times 10^{5}$ cells/well. Infected cells were seeded in 24 well plates and incubated with treated saliva at $15,10,5$ and $2.5 \mu \mathrm{g} / \mathrm{mL}$ iota-carrageenan. At $72 \mathrm{~h}$ post-infection, supernatants were harvested, incubated for $10 \mathrm{~min}$ at $95^{\circ} \mathrm{C}$ and analyzed via q-RT-PCR as described elsewhere. ${ }^{12,17}$

\section{Hemaggutionation Inhibiton Assay - HCoV OC43, PIV3 and Influenza Virus A HINIpdm09}

Virus and cell cultivation as well as antiviral activity assays for hCoV OC43, PIV3 and influenza virus A H1N1pdm09 were performed as described elsewhere. ${ }^{11}$ In short, two (hCoV OC43, PIV3) or four (influenza virus) hemagglutination units (HAU) were incubated with a semilogarithmic dilution series of test samples (starting at $3 \mu \mathrm{g} / \mathrm{mL}$ iotacarrageenan (hCoV OC43) or $30 \mu \mathrm{g} / \mathrm{mL}$ (influenza virus A H1N1pdm09, PIV3) for $10 \mathrm{~min}$ at room temperature. A suspension of chicken red blood cells was added to each well to allow hemagglutination (HA) of RBC by the virus for $1.5 \mathrm{~h}$ at $4^{\circ} \mathrm{C}$. At the time point of assay evaluation, control RBC in the absence of an antiviral were fully agglutinated by the virus, whereas inhibition of hemagglutination could be observed in the presence of treated saliva down to a certain concentration (MIC).

\section{Control Compounds}

Iota-carrageenan was purchased from Dupont former FMC Biopolymers (both Philadelphia, PA). The dry polymer powders were dissolved in cell culture water (B Braun Melsungen AG, Germany) to a final iota-carrageenan concentration of $2.4 \mathrm{mg} / \mathrm{mL}$ containing $0.5 \% \mathrm{NaCl}$ (Merck KGA, Germany). This stock solution was sterile filtered through a $0.22 \mathrm{~mm}$ filter (Sarstedt, Germany) and stored at $4^{\circ} \mathrm{C}$ until use. Iota-carrageenan was used as a reference in all assays. The quality of iota-carrageenan was analyzed via ${ }^{1} \mathrm{HNMR}$, which confirmed an iota-carrageenan content of $92.5 \%$ and minor quantities of kappa- and nucarrageenan.

\section{Safety}

No adverse events were recorded during the trial. Saliva of four subjects contained traces of blood due to previously unrecognized periodontitis. These saliva samples were omitted from analysis.

\section{Statistical Analysis}

The primary objective was to determine whether the mean iota-carrageenan concentration in saliva during sucking an iota-carrageenan containing lozenge reached published $\mathrm{IC}_{90}$ values for $\mathrm{hRV} 1 \mathrm{a}$ and $\mathrm{hRV} 8(\sim 5 \mu \mathrm{g} / \mathrm{mL}$ iotacarrageenan). ${ }^{9}$

The null hypothesis is defined as: Mean iota-carrageenan concentration $(\mu \mathrm{g} / \mathrm{mL}$; baseline corrected) in saliva during sucking an iota-carrageenan containing lozenge $<$ published $\mathrm{IC}_{90}$ values of $\mathrm{hRV} 1 \mathrm{a}(5 \mu \mathrm{g} / \mathrm{mL}$ iota-carrageenan) and hRV8 (5 $\mu \mathrm{g} / \mathrm{mL}$ iota-carrageenan). ${ }^{9}$

The alternative hypothesis is defined as: Mean iotacarrageenan concentration $(\mu \mathrm{g} / \mathrm{mL}$; baseline corrected) in saliva during sucking an iota-carrageenan containing lozenge $\geq$ published $\mathrm{IC}_{90}$ values of hRV1a $(5 \mu \mathrm{g} / \mathrm{mL}$ iota-carrageenan) and hRV8 $(5 \mu \mathrm{g} / \mathrm{mL}$ iota-carrageenan $) .{ }^{9}$

Each of both co-primary endpoints was tested at significance level of 0.025 (one-sided). Study success was defined by a positive outcome of all endpoints thus no adjustment for multiple testing was required. By means of Kolmogorov-Smirnov-Lilliefors-Tests normality assumption of the data was proved. In case of normality, a onesample $t$-test was applied to test the null hypothesis; otherwise, a Wilcoxon signed-rank test was used at an alpha level of 0.025 (one-sided). In addition to p-values, the mean iota-carrageenan concentration was supported by $95 \%$ confidence intervals.

The secondary objectives were to determine whether the mean iota-carrageenan concentration in saliva $(\mu \mathrm{g} / \mathrm{mL}$; base line corrected) during sucking an iota-carrageenan containing lozenge reached the respective $\mathrm{IC}_{90} / \mathrm{MIC}$ values (paired t-tests or Wilcoxon signed-rank tests) of the clinical saliva samples against hRV1a, hRV8, hCoV OC43, influenza virus A H1N1n, PIV3, coxsackievirus A10 and SARS-CoV-2.

Furthermore, all assessed variables were evaluated using appropriate descriptive methods. Quantitative variables were described with the number of non-missing values, mean, standard deviation (SD), median, and 
minimum/maximum values. Qualitative values were described with the number and percentages of subjects with each qualitative characteristic. Missing values were not included in the calculation of percentages.

\section{Results}

\section{Study Population}

Thirty-one volunteers were screened, included and completed the study. Four of 31 native saliva samples contained traces of blood due to gum bleeding. Therefore, samples from these subjects had to be excluded from the efficacy analysis. Hence, the safety population comprises 31 subjects, whereas the per protocol (PP) population consists of 27 subjects. Demographic data of all volunteers $(n=31)$ show that $67.7 \%$ of the probands were female and $32.3 \%$ were male. The median age was $52.5( \pm 13.7)$ years, the mean body mass index was $27.06( \pm 5.9) \mathrm{kg} / \mathrm{m}^{2}$.

\section{Results of Statistical Evaluation of the Primary Efficacy Variable - Baseline Corrected lota-Carrageenan Concentration}

Since the assumption of normality for iota-carrageenan values was accepted as assessed by a Kolmogorov-Smirnov Test $(\mathrm{p}=0.71)$, a one sample $t$-test was computed to assess whether the baseline corrected mean iota-carrageenan concentration was different to the published $\mathrm{IC}_{90}$ values for hRV1a and hRV8 $(5 \mu \mathrm{g} / \mathrm{mL}) .{ }^{9}$ The pooled native saliva samples gave an unspecific signal corresponding to 44.40 to $89.27 \mu \mathrm{g} / \mathrm{mL}$ iotacarrageenan; a value by which the iota-carrageenan concentration of each treated samples was reduced to avoid its overestimation. The baseline-corrected mean iota-carrageenan concentration was $667.8 \mu \mathrm{g} / \mathrm{mL}$ (Table 1 and Figure 2) and thus statistically significantly higher than the published $\mathrm{IC}_{90}$ of both hRV1a and hRV8 $(5 \mu \mathrm{g} / \mathrm{mL}, \mathrm{p}<0.001,95 \%$ CI [581.5; 754.1]). Thus, the mean iota-carrageenan concentration present in the treated saliva samples exceeds the iota-carrageenan concentration that is necessary to reduce rhinoviral replication by $90 \%$ by more than 133.6 -fold $95 \%$ CI $[116.3 ; 150.8]$. The lowest concentration found in the treated saliva samples was $321.3 \mu \mathrm{g} / \mathrm{mL}$. Even this concentration exceeded published
$\mathrm{IC}_{90}$ values by 64.3 -fold. These results clearly show that the primary endpoint was met.

\section{Results of Statistical Evaluation of the Secondary Efficacy Variables}

For evaluation of the secondary objectives the specific antiviral activity of the treated saliva samples (expressed as $\mathrm{IC}_{90} / \mathrm{MIC}$ values) was determined in vitro as described in the Material \& Methods section (Table 2).

These values were then compared to the mean iota-carrageenan concentration of the clinical saliva samples ( 667.8 $\mu \mathrm{g} / \mathrm{mL}$ ). The assumption of normality for the difference of iota-carrageenan concentrations and the respective mean $\mathrm{IC}_{90}$ or minimum inhibitory concentration (MIC) values was valid as assessed by Kolmogorov-Smirnov Tests ( $p>$ 0.05 for all cell assays). The mean baseline corrected iotacarrageenan concentration was significantly higher than the $\mathrm{IC}_{90} / \mathrm{MIC}$ of hRV1a, hRV8, hCoV OC43, influenza virus A H1N1, coxsackievirus A10 and pandemic SARS-CoV-2 (Table 3 ) as computed by paired $t$-tests. Moreover, the iotacarrageenan concentration exceeded the $\mathrm{IC}_{90} / \mathrm{MIC}$ concentrations, depending on the virus, by 60 - to $30,350.9$-fold, indicating that significantly more iota-carrageenan than necessary to inactivate the tested viruses is present in the saliva after sucking an iota-carrageenan containing lozenge.

\section{Discussion}

Iota-carrageenan is a well-established antiviral with a strictly physical mode of action. ${ }^{9,10,19}$ It is neither absorbed nor metabolized and has therefore no pharmacological properties. ${ }^{19}$ Thus, the very presence of iota-carrageenan at the site of infection above a specific concentration, which varies from virus to virus and thus has to be determined specifically (eg. $\mathrm{IC}_{50}, \mathrm{IC}_{90}, \mathrm{MIC}$ ), attests its effectiveness.

The aim of the present study was to investigate whether the iota-carrageenan concentration in the saliva of subjects who sucked one iota-carrageenan lozenge was sufficient to inhibit the replication of the most predominant respiratory viruses causing common cold. The samples were tested for their antiviral effectiveness using virological standard methods (infectivity reduction assay,

Table I lota-Carrageenan Concentration and Statistical Parameters Assessed from Treated Saliva Samples

\begin{tabular}{|l|c|c|c|c|c|c|c|c|}
\hline & Mean & SD & Min & Max & QI & Median & Q3 & N \\
\hline Carrageenan conc. $[\mu \mathrm{g} / \mathrm{mL}]$ & 667.8 & 218.1 & 321.3 & 1048.1 & 475.1 & 729.2 & 854.7 & 27 \\
\hline
\end{tabular}

Abbreviations: Conc., concentration; SD, standard deviation; Min, minimum; Max, maximum; QI, first quartile; Q3, third quartile; N, population size. 


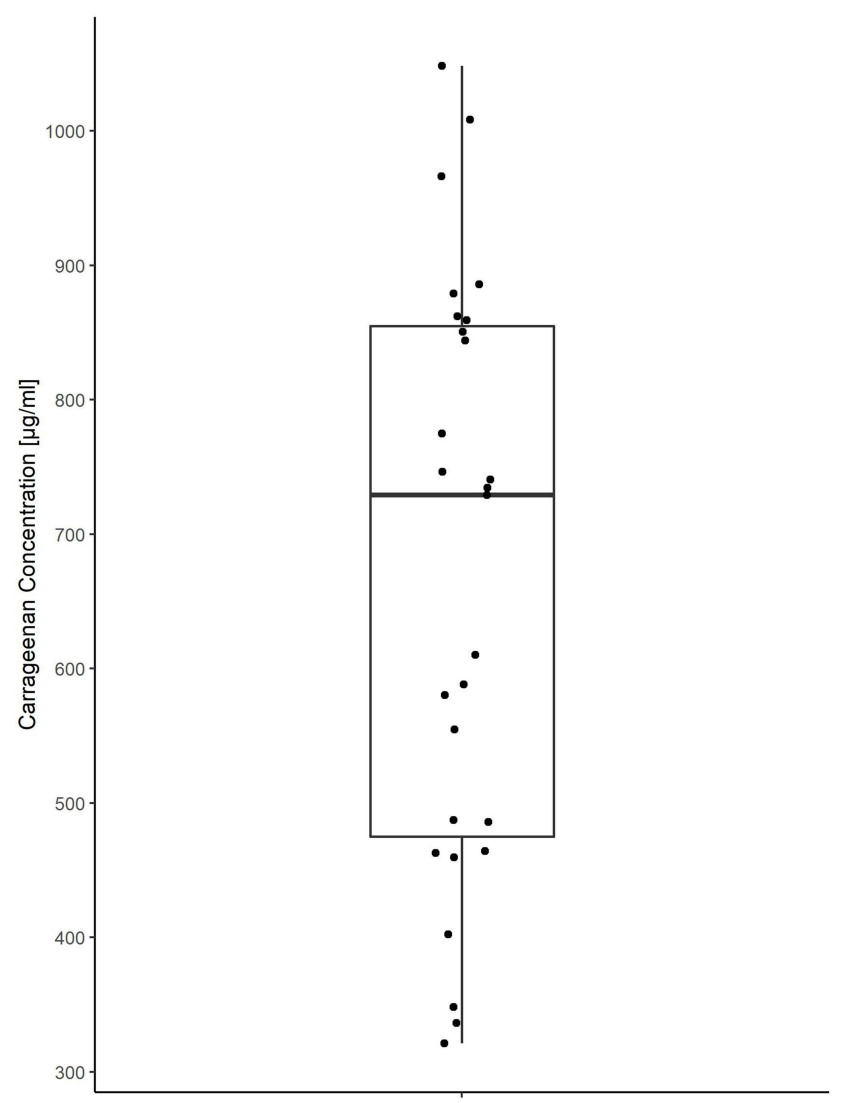

Figure 2 Boxplot of individual baseline corrected iota-carrageenan concentration.

hemagglutination inhibition assay) as published by the WHO and other healthcare institutions. ${ }^{20}$ These protocols were adapted for the herein used viruses and are described in detail in ${ }^{9-11}$ and in the Material and Methods section.

Our results clearly show that sucking an iota-carrageenan lozenge releases sufficient iota-carrageenan into the saliva to neutralize the most common respiratory viruses and even
SARS-CoV-2. First, we determined the iota-carrageenan concentration in saliva collected during sucking, showing that it exceeds the necessary published concentration of iota-carrageenan to inhibit hRV1a and hRV8 significantly ( $p<0.001)$. Second, we assessed the specific inhibitory effect of each of the treated saliva samples on several respiratory viruses: hRV1a and hRV8, hCoV OC43, influenza A virus H1N1n, coxsackievirus A10, PIV3, and SARS-CoV-2. Here, the mean iota-concentration exceeded the concentration needed to inhibit $90 \%$ of viral replication by 82 or 464 -fold in case of hRV1a or hRV8, by 1324-, 60- and 10,154-fold in case of influenza virus A H1N1n, coxsackievirus A10, and by PIV3, respectively. The effect was most pronounced with hCoV OC43, where the $\mathrm{IC}_{90}$ was exceeded by 30,351 -fold $(\mathrm{p}<0.001)$. No adverse events were recorded during the trial.

These findings are in good agreement with two clinical studies investigating the effect of an iota-carrageenan nasal spray in early common cold patients. ${ }^{4,6}$ The authors showed a significant antiviral efficacy in the virus positive population as well as in all three virus subgroups, eg, hRV, $\mathrm{hCoV}$, influenza virus A. The highest effectiveness was observed in human corona virus-infected patients, where the reduction of disease duration was more than 3 days, and the number of relapses was three times less in iotacarrageenan treated coronavirus infected patients compared to control patients. ${ }^{5}$

Hemilä et al performed an independent meta-analysis with publicly available data from clinical studies with an iota-carrageenan containing nasal spray. $^{21}$ The authors attest the quality of the available clinical data as well as the broad effectiveness against influenza viruses and endemic coronaviruses. They further conclude that nasal

Table 2 Antiviral Effectiveness of the Saliva Samples Against Various Viruses. IC 90 and MIC in $(\mu \mathrm{g} / \mathrm{mL})$

\begin{tabular}{|l|c|c|c|c|c|c|c|}
\hline & $\begin{array}{c}\text { hRVIa } \\
\text { (IC90) }\end{array}$ & $\begin{array}{c}\text { hRV8 } \\
\text { (IC90) }\end{array}$ & $\begin{array}{c}\text { hCoV OC43 } \\
\text { (MIC) }\end{array}$ & $\begin{array}{c}\text { IV A HINIn } \\
\text { (MIC) }\end{array}$ & $\begin{array}{c}\text { Coxsackievirus AI0 } \\
\text { (IC90) }\end{array}$ & $\begin{array}{c}\text { PIV3 } \\
\text { (MIC) }\end{array}$ & $\begin{array}{c}\text { SARS-CoV-2 } \\
\text { (IC90) }\end{array}$ \\
\hline Mean IC $/$ /MIC & 9.5 & 2.38 & 0.025 & 0.66 & 14.79 & 0.07 & 7.46 \\
SD & 3.71 & 3.00 & 0.012 & 0.25 & 11.02 & 0.01 & 4.72 \\
Minimum & 4.76 & 0.69 & 0.007 & 0.24 & 5.97 & 0.02 & 1.47 \\
Lower quartile & 6.79 & 1.17 & 0.024 & 0.52 & 8.13 & 0.07 & 4.75 \\
Median & 8.98 & 1.36 & 0.024 & 0.80 & 8.74 & 0.07 & 5.49 \\
Upper quartile & 11.22 & 2.44 & 0.024 & 0.80 & 21.32 & 0.07 & 9.89 \\
Maximum & 18.9 & 16.57 & 0.081 & 0.80 & 49.19 & 0.07 & 22.56 \\
\hline
\end{tabular}

Notes: The respective viruses were incubated with a dilution series of the treated saliva samples before infection of a susceptible cell line. After assay determination antiviral effectiveness was determined as described in the material and methods section $(\mathrm{N}=27$ ). The reference (iota-carrageenan in $0.5 \% \mathrm{NaCl}$ ) showed an equal or highly similar $\mathrm{IC}_{90} / \mathrm{MIC}$ as the treated saliva samples in all assays, indicating that the antiviral activity of iota-carrageenan persists in the complex saliva matrix.

Abbreviations: IV, influenza virus; MIC, minimal inhibitory concentration; IC 90 , 90\% maximal inhibitory concentration; SD, standard deviation; N, population size. 
Table 3 Calculated Mean Difference/Mean Excess Factor for the lota-Carrageenan Concentration Detected in Treated Saliva to IC $90 /$ $\operatorname{MIC}(95 \% \mathrm{Cl})$

\begin{tabular}{|l|c|c|c|}
\hline Virus Tested & Mean Difference $(\boldsymbol{\mu g} / \mathbf{m L})$ & Mean x-Fold Excess & p-value \\
\hline hRVIa & $658.20(571.7 ; 744.7)$ & $81.8(64.9 ; 98.6)$ & $<0.001$ \\
hRV8 & $665.42(578.68 ; 752.16)$ & $463.2(353.8 ; 572.6$ & $<0.001$ \\
hCoV OC43 & $667.77(581.49 ; 754.06)$ & $30,350.9(22,909.8 ; 37,792.0)$ & $<0.001$ \\
IV A HINIn & $667.14(580.87 ; 753.42)$ & $1323.6(964.5 ; 1682.7)$ & $<0.001$ \\
Coxsackievirus AI0 & $653.01(568.07 ; 737.95)$ & $60.0(47.4 ; 72.6)$ & $<0.001$ \\
PIV3 & $667.7(581.4 ; 754.0)$ & $10,154.0(8563.0 ; 11,746.0)$ & $<0.001$ \\
SARS-CoV-2 & $660.3(573.9 ; 746.8)$ & $120.5(93.2 ; 147.8)$ & $<0.001$ \\
\hline
\end{tabular}

Notes: Carrageenan concentrations of the saliva samples were compared to the concentrations needed to inhibit at least $90 \%$ of virus binding/replication (IC 90 , MIC). $\mathrm{Cl}_{\mathrm{L}}$ and $\mathrm{Cl}_{U}$ are shown in brackets. $\mathrm{p}$-values from paired $t$-tests indicate statistical significance of the differences.

Abbreviations: $\mathrm{Cl}$, confidence interval; $\mathrm{Cl}_{\mathrm{L}}$, lower confidence interval; $\mathrm{Cl}_{U}$, upper confidence interval; IV, influenza virus.

carrageenan may also inhibit the new coronavirus SARSCoV-2. This assumption has meanwhile been confirmed in vitro ${ }^{12,16}$ and clinically ${ }^{8,14}$ and is also supported by the present study which reveals that replication competent SARS-CoV-2 is effectively neutralized when incubated with iota-carrageenan treated saliva. The mean iota-carrageenan concentration in the saliva samples exceeded the respective $\mathrm{IC}_{90}$ by 120.5 -fold $(\mathrm{p}<0.001)$. These data are in line with former in vitro experiments showing that iotacarrageenan inhibits cell entry of the SARS-CoV-2 spike pseudo typed lentivirus and replication of SARS-CoV-2 with an $\mathrm{IC}_{50}$ of $<2.6 \mu \mathrm{g} / \mathrm{mL}$ iota-carrageenan. ${ }^{12}$ Jang et al confirm the potent antiviral activity of carrageenan against SARS-CoV-2 in vitro and in vivo. ${ }^{22}$ Furthermore, Schuetz et al demonstrate that iota- and kappa- carrageenans suppress SARS-CoV-2 infection of TMPRSS2-42 Vero E6 cells and primary differentiated human airway epithelial cultures. The authors conclude that the application of carrageenan containing nasal and mouth sprays may reduce the risk of acquiring SARS-CoV-2 infection and may limit viral spread. ${ }^{16}$ Indeed, a multicenter, randomized, double-blinded, placebo-controlled trial assessing the prophylactical use of nasal iota-carrageenan in hospital personnel dedicated to caring for COVID-19 patients revealed significant efficacy in preventing SARS-CoV-2 infection. $^{8}$

The COVID-19 pandemic triggered the development of numerous oral and nasal sprays which claim to reduce viral infection in the nasal/oral cavity. A recent review identified 14 such sprays in development; many of these sprays are already marketed as medical devices in Europe. ${ }^{23}$ However, a closer look often reveals a lack of safety and efficacy data. Huijghebaert et al investigated a trypsin containing mouth spray (Viroprotect ${ }^{\circledR}$,
ColdZyme ${ }^{\circledR}$ ) for which it was claimed to prevent common cold and COVID-19. After thorough data analysis, the authors concluded that exogenous trypsin exposure might not only be ineffective, but might even trigger adverse effects on viral transmission, susceptibility/infection and mutation. $^{23}$ In contrast, no hRV escape mutants could be detected after 10 passages in the presence of low-level iota-carrageenan. $^{9}$ As iota-carrageenan non-specifically envelops the virus, thereby preventing interaction between virus and cellular surface, development of resistance due to the occurrence of escape mutants is unlikely. There are also lozenges on the market claiming antiviral activity. We tested a set of lozenges with Amylmetacresol/2,4-dichlorobenzyl alcohol or hexylresorcinol as active ingredient for their inhibitory effect on a set of common cold viruses and used iota-carrageenan containing lozenges as comparator. Interestingly, out of nine tested lozenges, only the iotacarrageenan containing one exerted fast, broad and comprehensive antiviral activity against all tested viruses. ${ }^{11}$ Safety and efficacy of intranasally applied iota-carrageenan have been demonstrated in several clinical trials in more than 600 children and adults. ${ }^{3-6,8}$ Additionally, postmarketing data of more than 19 million sold nasal sprays and 1.4 million sold packs of lozenges confirm its excellent safety profile.

In conclusion, this clinical study clearly demonstrates that sucking of a lozenge containing $10 \mathrm{mg}$ iota-carrageenan releases sufficient antivirally active iota-carrageenan into the saliva to neutralize the most abundant viruses causing common cold and sore throat $(\mathrm{hRV}$, endemic $\mathrm{hCoV}$, influenza virus, coxsackievirus, PIV) as well as pandemic SARS-CoV-2.

Complete neutralization is known to occur within 20 min and starts during sucking of the lozenge (mean 
dissolution time is $13 \mathrm{~min} 20 \mathrm{sec}) .{ }^{11}$ The iota-carrageenan containing salivary film then covers the surface of the oral cavity (saliva volume needed is about $2.147 \mathrm{~mL}$ as calculated from ${ }^{24}$ ) where neutralization is assumed to persist during the mean residence time of carrageenan at the mucosa, which is about four hours. ${ }^{17}$

This neutralization presumably leads not only to a reduction of infection, but also to a reduced transmissibility within a population and/or translocation to the lower respiratory tract, hereby potentially preventing more severe illnesses.

As iota-carrageenan exhibits broad antiviral activity, iota-carrageenan lozenges should be considered as an easily available first line-defense against circulating as well as newly emerging viruses to close the gap between virus identification and successful development of vaccines or specific antiviral medication.

\section{Data Sharing Statement}

Data related to this manuscript can be made available from the corresponding author upon reasonable request.

\section{Funding}

The research was partially funded by the Österreichische Forschungsförderungsgesellschaft (FFG, Austrian research promotion agency) - 880687 and the Deutsche Forschungsgemeinschaft (DFG, German Research Foundation) - 401821119/GRK2504 to US.

\section{Disclosure}

Martina Morokutti-Kurz, Nicole Unger-Manhart, Philipp Graf, Julia Kodnar are employed by Marinomed Biotech AG. Eva Prieschl-Grassauer and Andreas Grassauer are co-founder of Marinomed Biotech $\mathrm{AG}$ and inventor on patent \#WO2008067982 held by Marinomed Biotech AG that relates to the content of the manuscript. Markus Savli reports personal fees from Marinomed Biotech AG. Andreas Grassauer, Eva Prieschl-Grassauer and Martina Morokutti-Kurz are inventors of a patent submission related to the content of the manuscript; the number of this patent application is EP20186334. The authors report no other conflicts of interest in this work.

\section{References}

1. Denny FW Jr. The clinical impact of human respiratory virus infections. Am J Respir Crit Care Med. 1995;152(4 Pt 2):S4-S12. doi:10.1164/ajrccm/152.4_Pt_2.S4
2. Snell NJ. New treatments for viral respiratory tract infections opportunities and problems. J Antimicrob Chemother. 2001;47 (3):251-259. doi:10.1093/jac/47.3.251

3. Eccles R, Meier C, Jawad M, Weinmüllner R, Grassauer A, PrieschlGrassauer E. Efficacy and safety of an antiviral Iota-Carrageenan nasal spray: a randomized, double-blind, placebo-controlled exploratory study in volunteers with early symptoms of the common cold. Respir Res. 2010;11(1):108. doi:10.1186/1465-9921-11-108

4. Fazekas T, Eickhoff P, Pruckner N, et al. Lessons learned from a double-blind randomised placebo-controlled study with a iota-carrageenan nasal spray as medical device in children with acute symptoms of common cold. BMC Complement Altern Med. 2012;12(1):147. doi:10.1186/1472-6882-12-147

5. Koenighofer M, Lion T, Bodenteich A, et al. Carrageenan nasal spray in virus confirmed common cold: individual patient data analysis of two randomized controlled trials. Multidiscip Respir Med. 2014;9 (1):57. doi:10.1186/2049-6958-9-57

6. Ludwig M, Enzenhofer E, Schneider S, et al. Efficacy of a carrageenan nasal spray in patients with common cold: a randomized controlled trial. Respir Res. 2013;14(1):124. doi:10.1186/1465-9921-14-124

7. Eccles R, Winther B, Johnston SL, Robinson P, Trampisch M, Koelsch S. Efficacy and safety of iota-carrageenan nasal spray versus placebo in early treatment of the common cold in adults: the ICICC trial. Respir Res. 2015;16(1):121. doi:10.1186/s12931-015-0281-8

8. Figueroa JM, Lombardo M, Dogliotti A, et al. Efficacy of a nasal spray containing iota-carrageenan in the prophylaxis of COVID-19 in hospital personnel dedicated to patients care with COVID-19 disease a pragmatic multicenter, randomized, double-blind, placebo-controlled trial (CARR-COV-02). medRxiv. 2021. doi:10.1101/2021.04.13.21255409

9. Grassauer A, Weinmuellner R, Meier C, Pretsch A, PrieschlGrassauer E, Unger H. Iota-carrageenan is a potent inhibitor of rhinovirus infection. Virol J. 2008;5(1):107. doi:10.1186/1743422X-5-107

10. Leibbrandt A, Meier C, König-Schuster M, et al. Iota-carrageenan is a potent inhibitor of influenza A virus infection. PLoS One. 2010;5 (12):e14320. doi:10.1371/journal.pone.0014320

11. Morokutti-Kurz M, Graf C, Prieschl-Grassauer E. Amylmetacresol/ 2,4-dichlorobenzyl alcohol, hexylresorcinol, or carrageenan lozenges as active treatments for sore throat. Int J Gen Med. 2017;10:53-60. doi:10.2147/IJGM.S120665

12. Morokutti-Kurz M, Fröba M, Graf P, et al. Iota-carrageenan neutralizes SARS-CoV-2 and inhibits viral replication in vitro. PLoS One. 2021;16(2):e0237480. doi:10.1371/journal.pone.0237480

13. Yasir M, Goyal A, Bansal P, Sonthalia S, eds. StatPearls [Internet]. StatPearls Publishing; 2020.

14. Bansal S, Jonsson CB, Taylor SL, et al. Iota-carrageenan and xylitol inhibit SARS-CoV-2 in cell culture. bioRxiv. 2020. doi:10.1101/ 2020.08.19.225854

15. Charlton CL, Babady E, Ginocchio CC, et al. Practical guidance for clinical microbiology laboratories: viruses causing acute respiratory tract infections. Clin Microbiol Rev. 2019;32(1);e00042-e00118. doi:10.1128/CMR.00042-18

16. Schütz D, Conzelmann C, Fois G, et al. Carrageenan containing over-the-counter nasal and oral sprays inhibit SARS-CoV-2 infection of airway epithelial cultures. Am J Physiol Lung Cell Mol Physiol. 2021;320(5):L750-L756. doi:10.1152/ajplung.00552.2020

17. Graf C, Bernkop-Schnürch A, Egyed A, Koller C, Prieschl-Grassauer E, Morokutti-Kurz M. Development of a nasal spray containing xylometazoline hydrochloride and iota-carrageenan for the symptomatic relief of nasal congestion caused by rhinitis and sinusitis. Int J Gen Med. 2018;11:275-283. doi:10.2147/IJGM.S167123

18. Große M, Ruetalo N, Businger R, et al. Evidence that quinine exhibits antiviral activity against SARS-CoV-2 infection in vitro. Preprints. 2020;2020070102. 
19. Eccles R. Iota-carrageenan as an antiviral treatment for the common cold. Open Virol J. 2020;14(1):9-15. doi:10.2174/1874357902014010009

20. World Health Organization.WHO Manual on Animal Influenza Diagnosis and Surveillance. Available from: https://www.who.int/ csr/resources/publications/influenza/whocdscsrncs20025rev.pdf. Accessed August 31, 2021.

21. Hemilä H, Chalker E. Carrageenan nasal spray may double the rate of recovery from coronavirus and influenza virus infections: re-analysis of randomized trial data. Pharmacol Res Perspect. 2020;9(4):e00810

22. Jang Y, Shin H, Lee MK, et al. Antiviral activity of lambda-carrageenan against influenza viruses and severe acute respiratory syndrome coronavirus 2. Sci Rep. 2021;11(1):821. doi:10.1038/s41598-020-80896-9
23. Huijghebaert S, Vanham G, van Winckel M, Allegaert K. Does trypsin oral spray (Viruprotect ${ }^{\mathbb{B}} /$ ColdZyme $^{\mathbb{B}}$ ) protect against COVID-19 and common colds or induce mutation? Caveats in medical device regulations in the European union. Int $J$ Environ Res Public Health. 2021;18(10):5066. doi:10.3390/ijerph18105066

24. Collins LM, Dawes C. The surface area of the adult human mouth and thickness of the salivary film covering the teeth and oral mucosa. $J$ Dent Res. 1987;66(8):1300-1302. doi:10.1177/0022034587 0660080201
International Journal of General Medicine

\section{Publish your work in this journal}

The International Journal of General Medicine is an international, peer-reviewed open-access journal that focuses on general and internal medicine, pathogenesis, epidemiology, diagnosis, monitoring and treatment protocols. The journal is characterized by the rapid reporting of reviews, original research and clinical studies
Dovepress

across all disease areas. The manuscript management system is completely online and includes a very quick and fair peer-review system, which is all easy to use. Visit http://www.dovepress.com/ testimonials.php to read real quotes from published authors. 